\title{
Expression of TAU in insulin-secreting cells and its interaction with the calcium-binding protein secretagogin
}

\author{
Magdalena Maj ${ }^{1}$, Wolfgang Gartner ${ }^{1,3}$, Aysegul Ilhan ${ }^{1}$, Dashurie Neziri ${ }^{1}$, Johannes Attems ${ }^{2}$ \\ and Ludwig Wagner ${ }^{1}$ \\ ${ }^{1}$ Department of Medicine III, Medical University of Vienna, Währinger Gürtel 18-20, 1090 Vienna, Austria \\ ${ }^{2}$ Institute for Aging and Health, Newcastle University, Newcastle upon Tyne NE4 5PL, UK \\ ${ }^{3}$ St Vincent Hospital, 1060 Vienna, Austria \\ (Correspondence should be addressed to L Wagner; Email: ludwig.wagner@meduniwien.at)
}

\begin{abstract}
Tauopathies have been associated with Alzheimer's disease (AD), which frequently manifests together with diabetes mellitus type 2. Calcium-binding proteins such as the recently identified secretagogin (SCGN) might exert protective effects. As pancreatic $\beta$-cells and neurons share common electrophysiological properties, we investigated the appearance of TAU (listed as MAPT in the HUGO and MGI Databases) protein at the islets of Langerhans and $\beta$-cellderived cell lines which highly express the neuroendocrinespecific protein SCGN. Six predominant TAU isoforms could be identified by immunoblotting, which formed TAU deposits detectable by immunofluorescence and sarkosylinsoluble pellets. Using GST-SCGN pull-down assays, a calcium-dependent SCGN-TAU interaction was found. In this line, sucrose density gradient fractionation and differential

ultracentrifugation studies of TAU and SCGN revealed co-appearance of both proteins. Co-localization of TAU and SCGN within insulinoma cells and islets of Langerhans mainly restricted to insulin-positive $\beta$-cells was demonstrated by confocal microscopy. Motivated by these findings, we looked if SCGN overexpression could exert protective function on Rin-5F cells, which showed differences in TAU levels. Testing the vulnerability of Rin-5F clones by MTT assay, we revealed that high TAU levels going along with highest TAU aggregates could not be antagonized by high levels of SCGN protein. Our findings demonstrated for the first time the association of TAU and the calcium-binding protein SCGN and support earlier results implicating that $\beta$-cells might represent an extra cerebral site of tauopathy. Journal of Endocrinology (2010) 205, 25-36
\end{abstract}

\section{Introduction}

Tauopathy has been associated with neurodegenerative diseases, cognitive disorders and, in this context most importantly, Alzheimer's disease (AD; Kosik et al. 1986, Goedert et al. 1988). Therefore, the TAU (listed as MAPT in the HUGO and MGI Databases) protein has attracted a lot of interest. TAU is important for microtubule dynamics (Goedert \& Jakes 1990) and axoplasmic transport (LaPointe et al. 2009). AD is characterized by the occurrence of TAU deposits and formation of neurofibrillary tangles. In the protein deposits characterizing neurons of AD, TAU occurs in hyperphosphorylated (P-TAU) states (Delacourte \& Defossez 1986, Grundke-Iqbal et al. 1986, Greenberg \& Davies 1990). P-TAU has been shown to be the key mediator of neurodegeneration and cognitive impairment (Santacruz et al. 2005). P-TAU disrupts neuronal microtubules (Alonso et al. 2001) followed by synaptic pathology and neuronal apoptosis. One hypothesis for cellular pathology of fibrillary tangles formation suggests a continuous intracellular hypoglycaemic state resulting in decreased O-GlcNAcylation of
TAU and subsequent exposition to kinases leading to hyperphosphorylation (Dias \& Hart 2007). However, till now, the exact cellular processes finally leading to cytotoxic effects and influencing factors are still unknown.

Neurons and neuroendocrine cells share several characteristics with respect to protein expression patterns, electrophysiological properties and pathophysiological processes (Tischler 2002). Similar to neurodegenerative diseases, $\beta$-cells of individuals suffering from type 2 diabetes are characterized by accumulation of intracellular islet amyloid peptide (IAPP) deposits ( Janson et al. 2004, Miklossy et al. 2008). In addition to IAPP, the presence of P-TAU has been demonstrated recently in pancreatic islets of individuals suffering from diabetes mellitus type 2 (Miklossy et al. 1999, 2008, Janson et al. 2004). In analogy to neurons of the central nervous system (CNS), these protein deposits seem to exert cytotoxic effects to $\beta$-cells (Lorenzo et al. 1994, O'Brien et al. 1995, Schubert et al. 1995, Janson et al. 1999). The co-appearance of islet amyloid and neuritic plaques in the brain highlights the analogy between $\mathrm{AD}$ and type 2 diabetes at the cellular level, which is reflected by epidemiological studies (Janson et al. 2004, Ristow 2004). 
A major characteristic shared by neurons and $\beta$-cells is the excitability, leading via an increase in intracellular calcium ions to exocytosis. Several calcium-binding proteins have been shown to be responsible for transmission of these calcium signals. In light of the functional importance of the calcium homoeostasis for exocytosis in a pulsatile mode and its impairment in the course of type 2 diabetes (O'Rahilly et al. 1988), the involvement of TAU and calcium-binding proteins in these processes has to be highlighted.

In this respect, the neuroprotective effect of calciumbinding proteins has to be stressed. Calretinin-immunoreactive neurons seem to be unaffected in $\mathrm{AD}$, and calbindin seems to exert neuroprotective effects in ageing neurons (Hof et al. 1993, Greene et al. 2001). Another point in this direction is the recently described resistance of hippocampal neurons expressing the calcium-binding protein secretagogin (SCGN) to neurodegeneration (Attems et al. 2008). The parallel involvement of calcium-binding proteins and TAU, which is suggested by these data, was underlined by the recently described interaction of a novel EF-hand calcium-binding protein and TAU in the CNS (Vega et al. 2008).

SCGN is a calcium-binding protein with similarities to calmodulin and calbindin-D28K, which is strongly expressed in pancreatic $\beta$-cells, other neuroendocrine cells and distinct neurons of the CNS (Wagner et al. 2000, Gartner et al. 2001, Cras-Meneur et al. 2004, Attems et al. 2007, Rogstam et al. 2007). SCGN was demonstrated to exert effects on secretion control and transcription processes in $\beta$-cells. Based on these findings and on recent structural analysis, SCGN was classified rather as calcium sensor than as calcium buffer (Wagner et al. 2000, Gartner et al. 2007, Rogstam et al. 2007).

To address this subject, we investigated the appearance of TAU isoforms in $\beta$-cells using a rodent insulinoma cell model. Furthermore, we investigated the biophysical states of TAU using confocal microscopy, ultracentrifugation and detergent treatment to reveal its resemblance to tauopathy in $\mathrm{AD}$.

Motivated by the established protective potential of calcium-binding proteins, we investigated the parallel expression of neuroendocrine-specific SCGN and TAU in insulin-secreting cells and tested whether overexpression could influence features of tauopathy. We additionally analysed the interaction capacities of both proteins and present its calcium dependence.

\section{Materials and Methods}

\section{Antibodies}

The polyclonal rabbit anti-human TAU (Cat. no. A 0024) was purchased from DakoCytomation (Denmark, Glostrup), the anti-TAU (3-repeat isoform RD3; Cat. no. 05-803) from Upstate Cell Signaling Solution (Lake Placid, NY, USA), the anti-human PHF-TAU mAb (clone AT8; Lot. no. FK 93121) from Pierce (Rockford, IL, USA), the mouse monoclonal anti-SCGN D24 Ab (Cat. no. MAB5622, Chemicon,
Temecula, CA, USA), the mouse monoclonal anti- $\beta$-actin (AC-15; Cat. no. NB600-501) from Novus Biologicals (Littleton, CO, USA), the monoclonal anti-SNAP-25 (Cat. no. S5187) from Sigma-Aldrich, the monoclonal anti$\alpha$-tubulin (Cat. no. T9026) from Sigma-Aldrich, the mouse monoclonal anti-rat insulin D3E7 (Cat. no. RDITRK2IP10-D3E7, Research Diagnostics Inc., Flanders, NJ, USA), the peroxidase-conjugated goat anti-mouse immunoglobulins (Cat. no. P 0447) and the peroxidase-conjugated goat anti-rabbit immunoglobulins (Cat. no. P 0448) from DakoCytomation. The rabbit anti-SCGN antibody was generated against recombinantly produced SCGN protein as described in previous work (Wagner et al. 2000, Gartner et al. 2001). The Alexa Fluor $488 \mathrm{~F}\left(\mathrm{ab}^{\prime}\right)_{2}$ fragment of goat antirabbit $\operatorname{IgG}$ (Cat. no. A 11070) was obtained from Invitrogen, TO-PRO from Invitrogen (Cat. no. T-3605), and the goat anti-mouse tetramethylrhodamine isothiocyanate-labeled affinity-purified $\mathrm{F}\left(\mathrm{ab}^{\prime}\right)_{2}$ fragment from Accurate Chemical and Scientific (Westbury, NY, USA).

\section{Cell culture conditions}

Rin-5F clones were generated according to the method as described earlier (Wagner et al. 2000). In brief, Rin-5F cells were transfected with SCGN encoding plasmid (SCGNpZeoSV2) and selected using Zeocin (Invitrogen).

Rin-5F and INS-1E cells were cultured in RPMI 1640 medium supplemented with $10 \%$ foetal bovine serum, $2 \mathrm{mM}$ L-glutamine, $100 \mathrm{IU} / \mathrm{ml}$ penicillin, $10 \mu \mathrm{g} / \mathrm{ml}$ streptomycin and $50 \mu \mathrm{M}$ 2-mercaptoethanol (complete culture medium) in a humidified atmosphere with $5 \% \mathrm{CO}_{2}$ at $37^{\circ} \mathrm{C}$. Cell cultures were passaged every 3 days using trypsin $(0 \cdot 25 \%)$.

In order to mimic diabetic conditions, we used sera from diabetic patients and non-diabetic individuals. The complete culture medium was supplemented with $10 \%$ of these human sera, and individual clones were incubated using the supplemented culture media for $48 \mathrm{~h}$. Rin-5F insulinoma cell clones were then further processed by FACS analysis for intracellular TAU staining as described below. In parallel, mRNA was isolated and tested for TAU transcription using TaqMan probe-based TAU RT-qPCR as described below. Additionally, cellular protein was isolated and analysed for changes in TAU level by immunoblotting as described below.

\section{Preparation of cell lysates}

After washing the cell monolayer with PBS, cells were scraped from the culture dish into lysis buffer. The lysis buffer consisted of Tween 20 substituted (0.1\%) PBS (TPBS) supplemented with the following protease inhibitors: $1 \mathrm{mM}$ phenylmethylsulphonyl fluoride (PMSF; Sigma; Cat. no. P-7626) and $10 \mu \mathrm{g} / \mathrm{ml}$ aprotinin (Sigma; Cat. no. A6103). The resultant lysate was passed once through a $24 \mathrm{G}$ needle and centrifuged at $13000 \mathrm{~g}$. The remaining supernatant was either snap frozen in liquid nitrogen and stored at $-80{ }^{\circ} \mathrm{C}$, or immediately used. 
As an alternative, the cell monolayer was washed once with PBS and then scraped into $8 \mathrm{M}$ urea containing $1 \%$ 2-mercaptoethanol. The cell lysate was centrifuged at $48000 \mathrm{~g}$ for $15 \mathrm{~min}$ at $4{ }^{\circ} \mathrm{C}$ in order to pellet DNA. The supernatant was subsequently boiled for $2 \mathrm{~min}$ in SDS sample buffer and loaded onto SDS-PAGE.

\section{Immunoblotting}

The cell lysate $(20 \mu \mathrm{g})$ and recombinant protein were loaded onto SDS-PAGE gels (8 or 12\%). Following electrophoretic separation, proteins were transferred onto nitrocellulose using a semi-dry blotting device. The membranes were blocked with 1:10 diluted blocking solution (10\% BSA diluent/ blocking solution, Cat. no. 50-61-01, KPL, Gaithersburg, $\mathrm{MD}$, USA) and were then serially exposed to the primary antibody (over night at $4{ }^{\circ} \mathrm{C}$ ) and the detection $\mathrm{Ab}$ (HRPconjugated goat anti-mouse or goat anti-rabbit; $50 \mathrm{~min}$ at room temperature $(\mathrm{RT})$ ). Antigen visualization was performed by chemoluminescence using the Lumi Imager F1 (Roche). Pictures were processed by PhotoShop Version 6. Signal intensities were densitometrically measured using the Lumi Analyst 3.1 software and set in relation to the housekeeping protein.

For phospho-TAU blotting, individual cell clones were collected and washed in ice-cold RPMI and lysed in Weinberg cell lysis buffer (50 mM Hepes, 0.5\% Nonidet P-40, $250 \mathrm{mM} \mathrm{NaCl}, 5 \mathrm{mM}$ EDTA, pH 7·0, $1 \mathrm{mM}$ PMSF, $1 \mathrm{mM}$ sodium orthovanadate, $50 \mathrm{mM} \beta$-glycerophosphate). The cell lysate was kept on ice for 10 min under frequent tapping and centrifuged at $13000 \mathrm{~g}$ at $4{ }^{\circ} \mathrm{C}$ for $10 \mathrm{~min}$. Lysates of equal cell numbers $\left(1 \times 10^{6}\right.$ cells $)$ from each clone were loaded onto SDS-PAGE and transferred onto PVDF membrane. After the blocking procedure as described above, blots were developed using AT8 mAb. For loading control, actin and the pan TAU blotting was used.

\section{FACS measurement}

After washing the cell monolayer with PBS, cells were trypsinized. A cell suspension $\left(1 \times 10^{6}\right)$ from each cell clone was transferred into FACS tubes (Screen Mates Tubes $1.4 \mathrm{ml}$, Cat. no. 4140, Thermo Scientific, Fisher, Vienna, Austria) in triplicates including a control for each clone. After washing cells with PBS, cells were fixed using $50 \mu$ fixation solution (Reagent A, Fix \& Perm, Cat. no. GAS-002, Szabo Scandic, Wien, Austria) for $25 \mathrm{~min}$ at RT. Following a PBS washing step, cells were permeabilized using $50 \mu \mathrm{l}$ permeabilization solution (Reagent B, Fix \& Perm, Cat. no. GAS-002, An Der Grub, Vienna, Austria) for $30 \mathrm{~min}$ at RT, subsequently adding $50 \mu \mathrm{l}$ primary antibody (anti-human TAU Ab, dilution 1:40) for $40 \mathrm{~min}$. After washing with PBS, cells were incubated for $50 \mathrm{~min}$ with $50 \mu \mathrm{l}$ secondary antibody (Alexa 488 goat anti rabbit, dilution 1:300). Following washing with PBS $(3 \times)$, FACS measurement was performed on Beckton Dickinson FacsCanto II. The mean fluorescence intensity was recorded in quadruplicate, and results were calculated as mean \pm s.D. Each experiment was carried out at least three times.

\section{Quantitative real-time PCR}

After pretreatment with DNase I (Invitrogen; 15 min, RT), $1 \mu \mathrm{g}$ total RNA was subjected to reverse transcription $(1 \mathrm{~h}$, $40^{\circ} \mathrm{C}$ ) using SuperScript II (Invitrogen). The synthesis reaction was stopped by heating $\left(15 \mathrm{~min}, 65^{\circ} \mathrm{C}\right)$.

cDNA was diluted 1:6 and used as template together with TaqMan TAU-specific probe (Applied Biosystems, Foster City, CA, USA) and $2 \times$ MasterMix (Applied Biosystems). Each sample was measured in duplicate in microtiter Optical 96-well Reaction Plate (Applied Biosystems). The PCR and data monitoring were carried out at the StepOnePlus Fast Real-Time PCR System (Applied Biosystems). Expression values were calculated according to the $\Delta \Delta C_{\mathrm{t}}$ method. As endogenous control, the TaqMan GAPDH probe (Applied Biosystems) has been used.

\section{Subcellular fractionation}

Rin-5F cell clones used for disruption and differential centrifugation were cultured for 2 days. In order to separate cellular compartments, a method developed by other research groups (Simpson et al. 1983, Albrektsen et al. 2001) was applied. After two washes in PBS, the cell monolayer was scraped into ice-cold PBS and washed once in sucrose holding buffer (SHB; $20 \mathrm{mM}$ Tris/ $\mathrm{HCl}, \mathrm{pH} 7 \cdot 4,1 \mathrm{mM}$ EDTA, $0.25 \mathrm{mM}$ sucrose) containing protease inhibitors (PMSF and aprotinin). All manipulations were carried out on ice. Cells were disrupted by pulling the cell suspension three times through a 24-gauge needle and twice through a 26-gauge needle. Cell nuclei were pelleted by centrifugation at $10000 \boldsymbol{g}(10 \mathrm{~min})$. The resultant post-nuclear supernatant was centrifuged for $20 \mathrm{~min}$ at $16000 \mathrm{~g}$ for obtaining a pellet consisting of mitochondria, peroxisomes (Mit/Per) and plasma membranes (PM). These were resuspended in $1.5 \mathrm{ml}$ SHB and overlaid onto a sucrose cushion buffer (1.12 M sucrose, $1 \mathrm{mM}$ EDTA, $20 \mathrm{mM}$ Tris/HCl, pH 7·4). After centrifugation at $101000 \mathrm{~g}$ for $25 \mathrm{~min}$, PM were taken from the interface, resuspended in SHB and centrifuged at $16000 \mathrm{~g}$ for $15 \mathrm{~min}$ for pelleting.

The supernatant obtained after pelleting mitochondria, peroxisomes and PM were centrifuged at $48000 \boldsymbol{g}(20 \mathrm{~min})$ resulting in a pellet of high-density microsomes (HD). This resultant supernatant underwent centrifugation at $212000 \mathrm{~g}$ for 70 min to yield a pellet containing low-density microsomes (LD). The supernatant of this final centrifugation step was taken as cytosol. Proteins in the pellet were redissolved in $8 \mathrm{M}$ urea. The protein content was evaluated by Bio-Rad protein assay. Equal quantity of protein $(15 \mu \mathrm{l})$ was loaded from each individual fraction onto an SDS-PAGE, blotted onto nitrocellulose and developed with anti-human pan-TAU Ab, anti-SCGN Ab, anti-SNAP-25 mAb and anti- $\alpha$-tubulin mAb. 


\section{TAU solubility assay}

The TAU solubility assay was performed as described recently (Khlistunova et al. 2006). Briefly, cells were scraped into $10 \mathrm{mM}$ Tris- $\mathrm{HCl}(\mathrm{pH} \mathrm{7} 4$ ) containing 0.8 M NaCl, $1 \mathrm{mM}$ EGTA and $10 \%$ sucrose. The resultant homogenate was spun for $20 \mathrm{~min}$ at $20000 \mathrm{~g}$. Subsequently, the pellet was re-homogenized and re-centrifuged at $20000 \mathrm{~g}$. Supernatants were combined and incubated for $1 \mathrm{~h}$ at $37^{\circ} \mathrm{C}$ in the presence of $1 \%$ of $N$-laurylsarcosinate. After a final centrifugation at $100000 \mathrm{~g}$ for $1 \mathrm{~h}$, the sarkosyl-insoluble pellet was dissolved in SDS-sample buffer containing 1.5\% 2-mercaptoethanol and was analysed by SDS-PAGE.

\section{Immunofluorescence}

Cytospin preparations of insulinoma cell culture cells as well as cryosections of pancreatic tissue (obtained from pancreatic tumour resection) were air dried and stained as described below or stored at $-20{ }^{\circ} \mathrm{C}$ wrapped in aluminium foil. Tumour tissue was obtained within a programme approved by the ethical committee of the Medical University of Vienna.

For immunostaining, frozen slides were brought to RT before unwrapping. Tissue sections were fixed in acetone, blocked with $100 \mu \mathrm{l}$ RPMI 1640 medium containing 10\% FCS followed by an incubation ( $4 \mathrm{~h}$, RT or over night at $4{ }^{\circ} \mathrm{C}$ ) with the following primary antibodies: the polyclonal rabbit anti-human TAU antibody, diluted 1:200 in PBS; or with the polyclonal, affinity purified rabbit anti-SCGN antibody, diluted 1:400 in PBS. For dual immunofluorescence, the rabbit polyclonal anti-human TAU $\mathrm{Ab}$ and the monoclonal mouse anti-SCGN mAb (clone D24, Millipore) were used as primary antibodies. As secondary antibodies, either the goat anti-mouse TRITC (diluted 1:200) or the goat anti-rabbit Alexa 488 (diluted 1:200) Abs were used (2 h). In dual colour analysis, both detection antibodies were used in parallel. For visualization of the nuclei, slides were counterstained with TO-PRO. Finally, the slides were mounted with Vectashield (Vector Laboratories, Burlingame, CA, USA). All incubations were performed in a moist chamber at room temperature. Between each incubation step, the slides were repeatedly washed for $10 \mathrm{~min}$ in PBS. Antigen visualization was performed using an Aristoplan fluorescence microscope (Leitz, Labortwert FS, Germany) or the Axiovert confocal microscope $200 \mathrm{M}$ (Zeiss, Jena, Germany). The images were processed using LSM 5 software (Zeiss).

\section{Generation of the human SCGN-GST recombinant fusion protein}

The coding region of the SCGN gene was cloned into the GST expression vector pGEX-1 $\lambda \mathrm{T}$ (Healthcare Biosciences, Sweden, Uppsala) as described earlier (Wagner et al. 2000). For protein production, the SCGN-GST encoding pGEX-1 $\lambda \mathrm{T}$ was expressed in Escherichia coli BL-21 according to the manufacture's instructions. Protein purification was performed using Glutathione Sepharose 4B (17-0756-01, Healthcare Biosciences). The GST fusion protein was liberated from the washed beads (PBS buffer) by thrombin cleavage.

\section{Pull-down assay}

GST-SCGN full-length or, for control, GST without fusion protein-loaded beads were incubated in precleared insulinoma cell lysate using non-denaturing lysis conditions (as described above) at $4{ }^{\circ} \mathrm{C}$ under constant rotation for $1 \mathrm{~h}$. After washing insulinoma cell lysate exposed beads, the bound protein was eluted by using EDTA (10 mM) containing washing buffer (TPBS). For subsequent analysis, the resultant protein eluate was boiled for $3 \mathrm{~min}$ and loaded onto SDS-PAGE. The gel was further processed as described in the immunoblotting section.

\section{MTT assay}

Equal numbers $\left(2 \times 10^{5}\right)$ of each cell clone were transferred into a 48-multiwell plate and incubated with complete medium or with $0 \cdot 1 \%$ BSA containing RPMI 1640 for $24 \mathrm{~h}$. Following this incubation period, the culture medium was removed and replaced with RPMI containing MTT (3-(4,5-dimethylthiazol-2-yl) 2,5-diphenytetrazoliumbromid); $500 \mu \mathrm{g} / \mathrm{ml})$. Cells were incubated for $60 \mathrm{~min}$, and after removal of supernatant, the resultant formazan dye was dissolved in $\mathrm{HCl}$ acidified $(50 \mathrm{mM})$ dimethyl sulphoxide (DMSO) containing SDS (10\% w/v). After a short incubation period $(2 \mathrm{~min})$, the colour intensity was evaluated by spectrophotometric measurement. Each experiment was carried out in tetraplicate and repeated at least three times on individual days.

\section{Results}

Co-expression of TAU and SCGN in $\beta$-cells and insulinoma cells

In order to answer the question of TAU presence in $\beta$-cells, we stained pancreatic tissue by dual colour fluorescence and demonstrated TAU-positive cells to co-localize with insulinpositive $\beta$-cells at the islets of Langerhans (Supplementary Figure 1, see section on supplementary data given at the end of this article). A negative control for TAU staining is included in the lower panel.

To evaluate the co-localization of TAU and SCGN, confocal immunofluorescence of islets of Langerhans (Fig. 1A, upper panel) and of human insulinoma cytospin preparations (Fig. 1A, lower panel) as well as of Rin-5F cytospin preparations was performed. This revealed considerable expression of TAU (green) in normal $\beta$-cells as well as in insulinoma cells (Fig. 1A). Expectedly, SCGN expression (red) was abundantly found in islet cells as well as in insulinoma cells (Fig. 1A). Using dual-colour visualization of individual antigens (TAU, green; SCGN, red), we found 

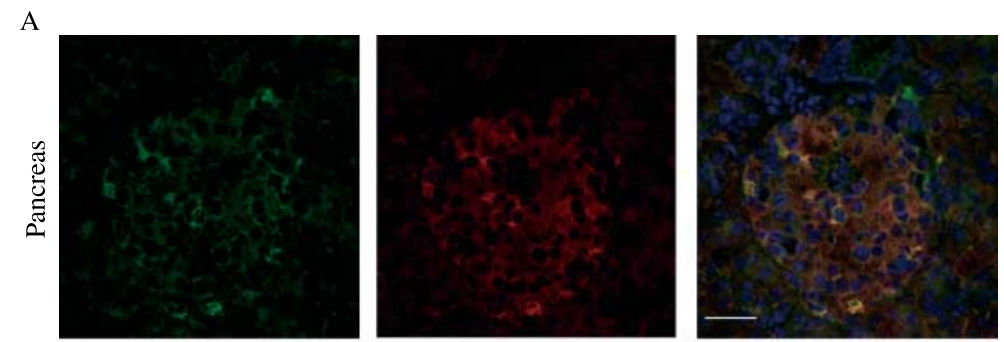

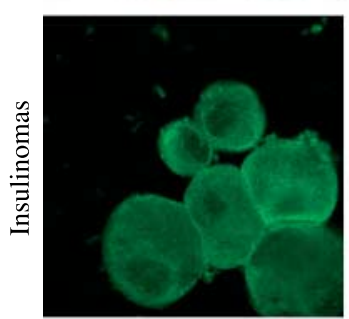

B
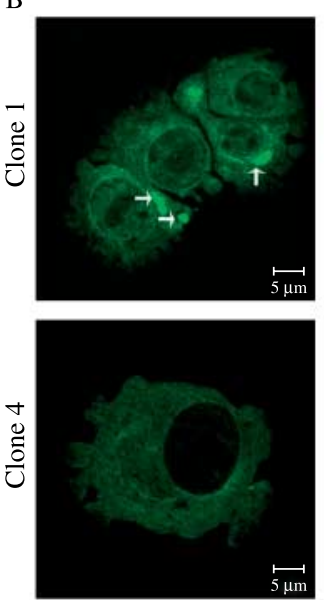

TAU

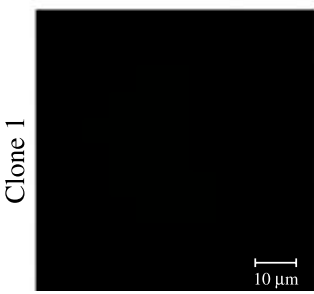

Control

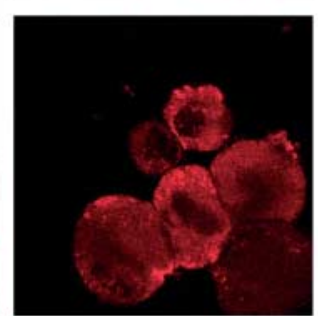

SCGN
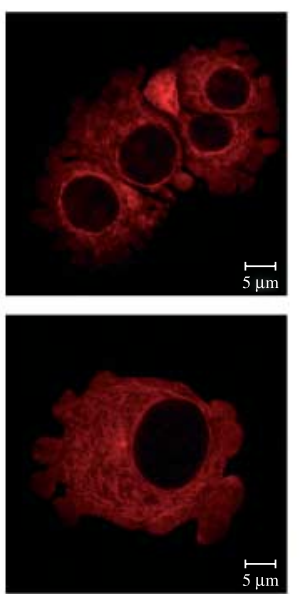

Tubulin

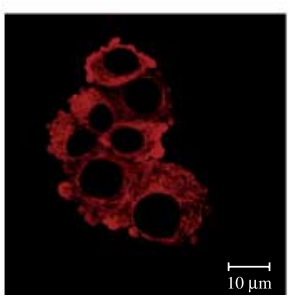

Tubulin

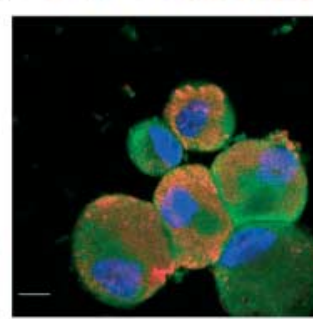

TAU/SCGN/TO-PRO
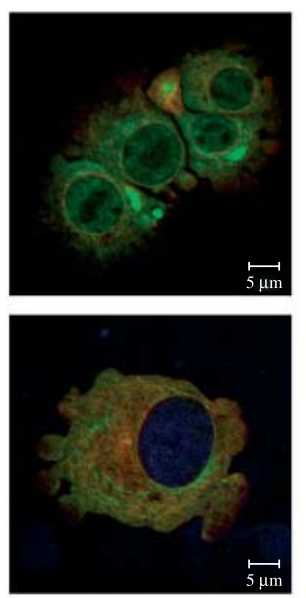

TAU/Tubulin

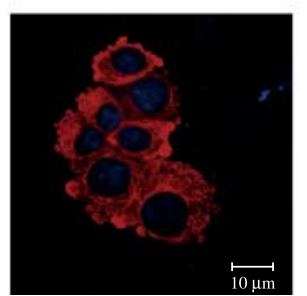

Tubulin/TO-PRO

Figure $1 \mathrm{TAU}$ immunofluorescene of pancreas, insulinomas and of the rat insulinoma cell line Rin-5F. (A) In pancreas (upper panel) and in insulinomas (lower panel), pan-TAU immunofluorescence staining revealed considerable TAU (green) expression. Co-immunostaining with SCGN a protein specific for islets of Langerhans (red) demonstrated co-localization of TAU and SCGN (yellow) within the individual cells. For nuclear staining, TO-PRO (blue) was used. Antigen visualization was performed by confocal microscopy (scale bar: upper panel $30 \mu \mathrm{m}$, lower panel $15 \mu \mathrm{m}$ ). (B) Using the pan-TAU Ab, we demonstrated strong TAU (green) expression in the rat insulinoma cell line Rin-5F and show cytoplasmic TAU aggregates (arrows) in clone 1 (upper panel). Dual-colour staining for TAU (green) and $\alpha$-tubulin (red) demonstrates Co-localization of both proteins to some degree (yellow coloration) within individual cells (upper and middle panel). For nuclear staining, TO-PRO (blue) was used. The lower panel demonstrates negative control for TAU staining using preimmune serum. Signal visualization was performed by confocal microscopy. 
a considerable extent of co-localization of both proteins (yellow) within the tested tissues (Fig. 1A). For nuclear staining, TO-PRO (blue) was used.

Interestingly, in Rin-5F cells, we detected occurrence of TAU deposits (arrow), which ranged from 5 to $8 \%$ in clone 1 (Fig. 1B, upper panel) to $1-2 \%$ in the other clones. To visualize the tubular distribution of TAU, we performed dualcolour confocal microscopy for TAU (green) versus $\boldsymbol{\alpha}$-tubulin (red) using clone 4. This demonstrated that in some areas
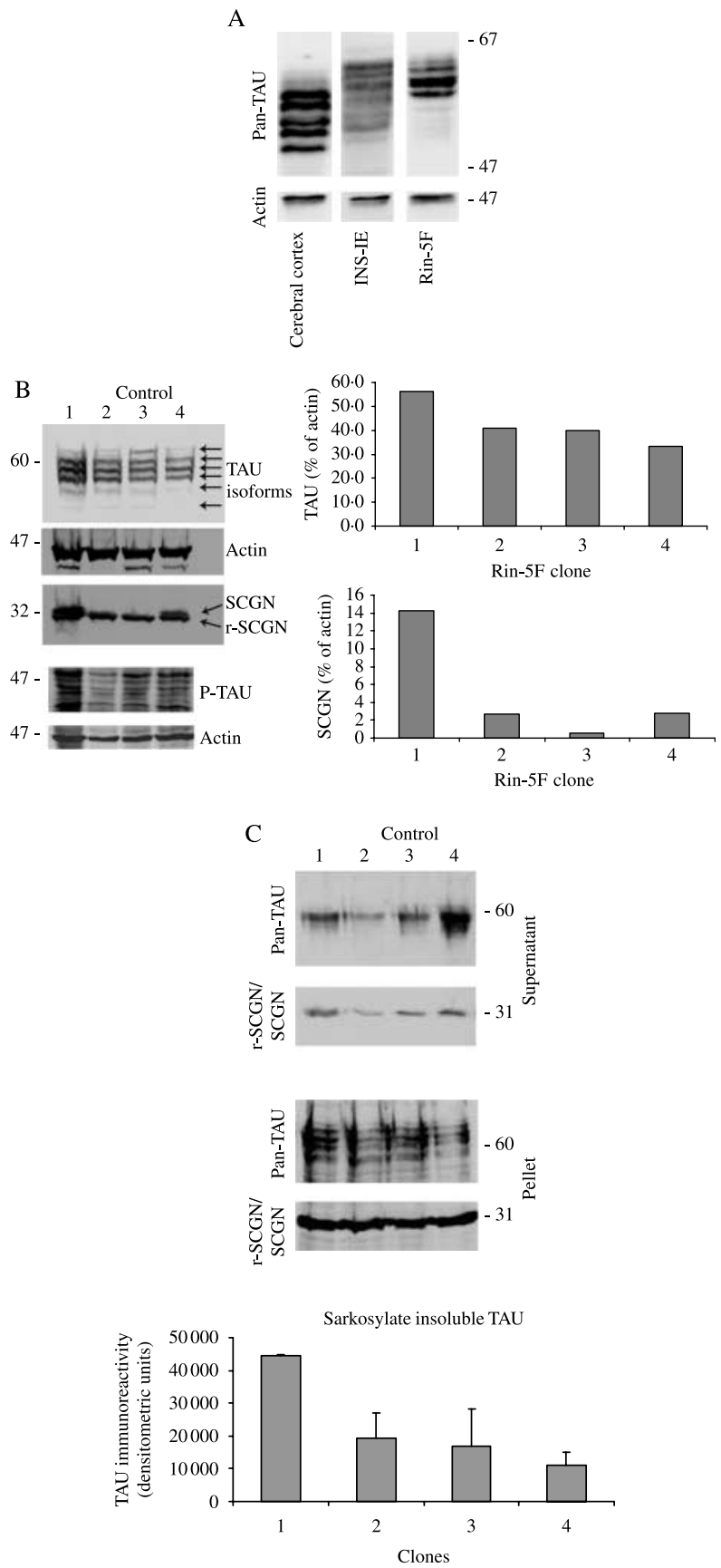

(centrosomes) TAU and tubulin co-localize (yellow coloration; Fig. 1B, middle panel). TAU negative control staining is demonstrated in the lower panel of Fig. 1B.

\section{Expression of TAU isoforms in rat insulinoma cell lines}

To investigate the presence of TAU isoforms in insulinoma cells, TAU immunoblotting was performed. Immunoblotting results confirm expression of TAU in rat insulinoma cells Rin5F and INS-1E (Fig. 2A). As a CNS-derived reference, cerebral cortex was included. This comparison intended to establish whether similar TAU isoforms occur in the CNS and pancreatic $\beta$-cells. Whereas in Rin-5F cells and INS-1E insulinoma cells, a comparable isoform pattern could be documented (Fig. 2A), and the two larger TAU variants seen in insulinomas were almost absent in CNS derived tissue lysate. In contrast, smaller variants known for CNS were much less abundant in insulinoma cell lysates. Nonetheless, TAU expression quantities in insulinoma cells did not reach the amount seen in the cerebral cortex.

Based on the co-expression of TAU and SCGN in insulinoma cells, we were interested if the expression extent of both proteins was correlated. For that purpose, the expression intensity of TAU was analysed in several Rin-5F clones characterized by different extents of SCGN expression. This revealed that the highest SCGN overexpression (clone 1) was paralleled by the highest TAU abundance (Fig. 2B). The other clones showed varying TAU and SCGN expression intensities. Notably, considerable amount of TAU was also present in the SCGN sham-transfectant clone 3 used as a control (Fig. 2B, lane 3). All clones expressed endogenous rat SCGN. TAU blots were densitometrically analysed, and TAU levels were set in relation to the housekeeping protein $\beta$-actin.

In order to evaluate the level of TAU phosphorylation, we performed P-TAU immunoblotting of the same cell clones and demonstrated high level of phosphorylation within all testes clones (Fig. 2B, lower panel). Note that for P-TAU

Figure 2 Expression of TAU isoforms in rat insulinoma cell lines. (A) Immunoblot analysis of INS-1E cells (lane 2) and of Rin-5F cells (lane 3) revealed strong TAU expression in the rat insulinoma cell lines. As reference, cerebral cortex (lane 1) was included. To confirm comparable protein loading, actin immunoblotting is presented in the lower panel. (B) To compare endogenous and recombinant SCGN ( $r$-SCGN) level with TAU protein level, immunoblotting of Rin-5F cell clones, clone 3 representing control (lane 1-4), using pan-TAU and SCGN-specific Abs was performed. Varying TAU and SCGN expression levels were detected. Actin was chosen as reference protein. P-TAU immunoblotting is demonstrated at the lower panel. Depicted is one representative figure out of three. For quantification, densitometric scanning was performed (right side of the corresponding blot). (C) Sarkosyl solubility of TAU in Rin-5F cells was determined by TAU solubility assay. This revealed the presence of sarkosyl-soluble (upper panel) and sarkosyl-insoluble TAU variants (lower panel, one representative experiment out of three is demonstrated). In parallel, SCGN was also found in both the pellet and the supernatant. The graph depicts the results of densitometric scanning (mean \pm s.D.) of three individually experiments performed on different days. 
immunoblotting, different cell lysis conditions had to be applied, which resulted in different banding pattern.

Independent of its isoforms, TAU occurs as sarkosyl-insoluble pellet especially in neurons of AD patients. To test Rin-5F cells for the presence of sarkosyl-insoluble TAU aggregates, a TAU solubility assay was performed. Expectedly, TAU was found in the sarkosyl-soluble fractions but also to a high amount in the sarkosyl-insoluble pellet (Fig. 2C). SCGN showed similar characteristics with respect to sarkosyl solubility. Clone 1, which was characterized by the highest SCGN expression, exhibited the highest amount of insoluble TAU aggregates.

Individual clones were tested for P-TAU by immunofluorescence. Highest P-TAU staining intensities were found in clone 1 followed by clone 4 (Fig. 3). In line with earlier studies performed on neuroblastoma cell lines, the immunofluorescence results demonstrated the presence of P-TAU in the cytoplasm but even to a stronger extent within the cell nucleus. In the cytosol, P-TAU exhibited a granular staining pattern. The nuclear presence was confirmed by subcellular fractionation as described further down.

\section{Rin-5F cells under type 2 diabetic conditions}

In order to investigate the influence of sera derived from patients suffering from diabetes mellitus type 2 on changes in TAU expression levels and isoform processing in Rin-5F cells, FACS analysis in parallel with RT-qPCR and western blotting of Rin-5F cells incubated with these sera was performed. We detected changes by FACS and RT-qPCR (increases); however, these did not reach the level of significance. In addition, we observed changes in cell morphology and growth pattern of insulinoma cell clones. In order to follow these findings, the influence of these conditions on TAU deposit formation was investigated by immunofluorescence microscopy. However, also in this direction, no significant difference could be observed.

\section{Intracellular distribution pattern of TAU in Rin-5F cells}

\section{Subcellular fractionation on a sucrose gradient}

To identify the intracellular TAU containing compartment and co-localized proteins, sucrose gradient fractionation of Rin-5F cells, and subsequent immunoblotting for pan-TAU,
$\boldsymbol{\alpha}$-TAU, $\boldsymbol{\alpha}$-tubulin, SNAP-25 and SCGN was performed (Fig. 4A). This revealed the presence of pan-TAU in all fractions with highest abundance in fractions 12-15, representing cytosolic compartments. Interestingly, P-TAU was also found in higher sucrose densities $(\sim 1-1.3 \mathrm{M})$ representative for membranous fractions and high molecular weight aggregates as well as at lower densities representative for cytosolic fractions $(\sim 0 \cdot 4-0 \cdot 3 \mathrm{M})$. Expectedly, $\boldsymbol{\alpha}$-tubulin was found to co-localize with cytosolic pan-TAU, whereas SNAP-25, a well-known member of the SNARE complex and marker for membranous cellular compartments, was found to co-localize with membrane-associated TAU or TAU aggregates. Co-appearance of SCGN/TAU was found in high sucrose densities as well as in cytosolic fractions (low densities).

Subcellular fractionation by ultracentrifugation To investigate these findings in more detail, ultracentrifugation of disrupted Rin-5F cells was performed. This resulted in the differentiation of six cellular compartments: LD, HD, Mit/Per, cytosol, nuclei and PM. The presence of TAU and SCGN was demonstrated by immunoblot analysis of the individual fractions (Fig. 4B). Interestingly, TAU protein was pelleted in the LD and HD fraction including degradation products, and was absent in cytosol. In cell nuclei, TAU protein was found at high quantity confirming immunofluorescence data. TAU within the nuclear fraction showed no degradation products. Consistent with sucrose gradient data, SCGN was also pelleted together with TAU in the LD and HD, and it was also found in high quantity in cytosol. Tubulin was detected in LD, cytosol and at lower quantity within the nuclei.

\section{TAU associated with SCGN in Rin-5F cells}

It has been shown that pyramidal neurons at the hippocampus expressing SCGN are greatly free of hyperphosphorylated TAU deposits (Attems et al. 2008), and TAU was shown to interact with other members of the EF-hand calcium-binding protein family in mouse brain (Vega et al. 2008). The calcium-binding protein SCGN has been demonstrated to be highly and specifically expressed within the islets of Langerhans (Wagner et al. 2000, Cras-Meneur et al. 2004). Therefore, TAU/SCGN

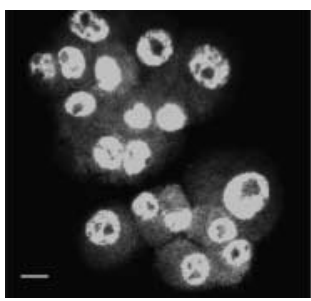

Clone 1

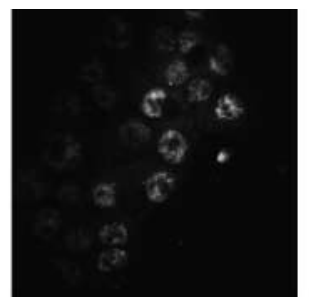

Clone 2

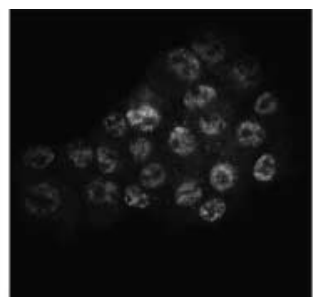

Clone 3 (control)

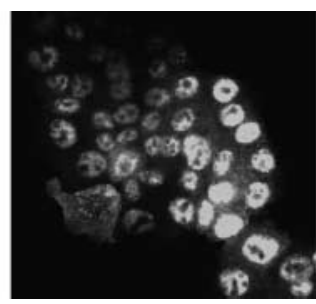

Clone 4

Figure 3 The presence of phospho-TAU in Rin-5F cells using AT8 mAb. To test for the presence of intracellular P-TAU, we performed immunofluorescence microscopy of various Rin-5F clones. This revealed the presence of P-TAU in each of the clones (scale bar: $10 \mu \mathrm{m}$ ). 


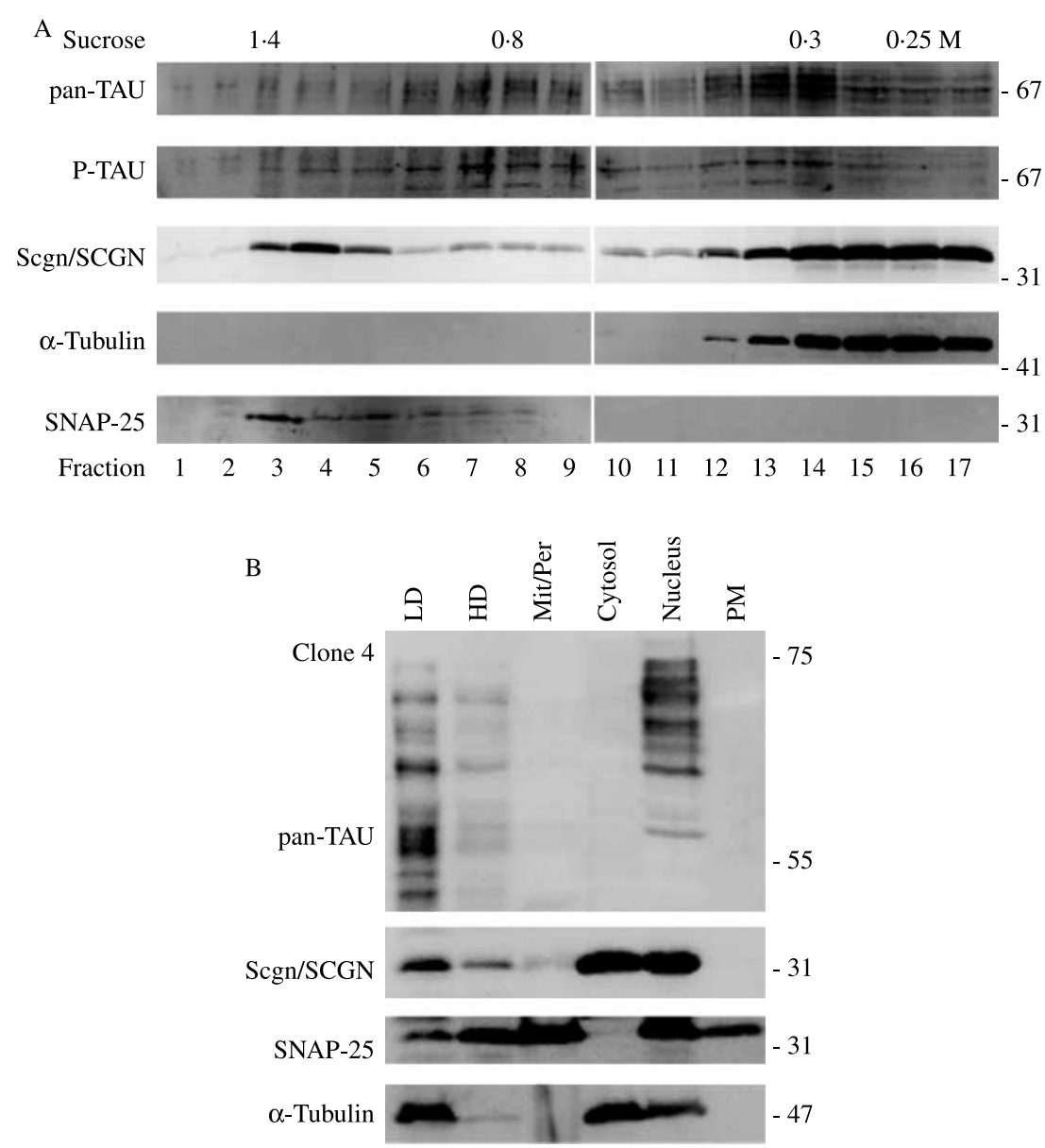

Figure 4 Intracellular TAU distribution pattern in Rin-5F cells. (A) Sucrose gradient fractionation. To analyse the intracellular TAU distribution, Rin-5F cell lysate was subjected to sucrose gradient fractionation and subsequent immunoblotting of the individual fractions. TAU isoforms (pan-TAU) as well as phosphorylated TAU (P-TAU) were found in the membranous as well as in the cytosolic fractions, with highest abundance of TAU in the cytosolic fractions. SNAP-25 immunoblotting was included to confirm the membranous compartments; $\alpha$-tubulin was included to confirm cytosplasmic compartments. In analogy to our immunofluorescence data, TAU was found to co-appear with SCGN. (B) Differential ultracentrifugation. TAU variants and degradation products resided in the low density microsomes (LD) and at lower quantity in the high density microsomes (HD). In addition, nuclear TAU, which had only marginal degradation products, was recovered. SCGN was detected in LD, HD, cytosol and nucleus. $\alpha$-Tubulin was detected in LD, cytosol, nucleus and in scant quantity in HD. SNAP-25 appeared at highest quantity in mitochondria/peroxisomes and nucleus as well as in LD, HD and PM.

interaction seemed plausible in neuroendocrine cells. For this purpose, the recombinant SCGN has been engineered as a GST fusion protein (Fig. 5A, upper panel, lane 1) and as a control GST alone was used (Fig. 5A, upper panel, lane2). The GST-SCGN beads were incubated with insulinoma cell lysate in parallel with GST only and after washing, the eluted proteins were loaded onto SDS-PAGE gels and blotted for the presence of TAU association. A single TAU-specific band could be observed at a molecular size of about $60 \mathrm{kDa}$ (Fig. 5A, lower panel, lane 1). No TAU binding was present in GST-loaded beads (Fig. 5A, lower panel, lane 2).
The striking observation that one single TAU isoform was interacting with SCGN led to ask a question whether this represents a $3 \mathrm{R}$ or $4 \mathrm{R}$ TAU isoform. Following this idea, we first developed the pull-down assay with a 3R TAU isoformspecific $\mathrm{mAb}$. This did not reveal any signal. In contrast, the pan-TAU-specific Ab detected the interacting TAU isoform (Fig. 5B, lane 3), suggesting that the SCGN-interacting TAU variant represents a $4 \mathrm{R}$ TAU. As a member of the EF-hand calcium-binding protein family, the functions of SCGN are mainly calcium dependent. To clarify if the TAU-SCGN interaction is calcium dependent, we included EDTA at 


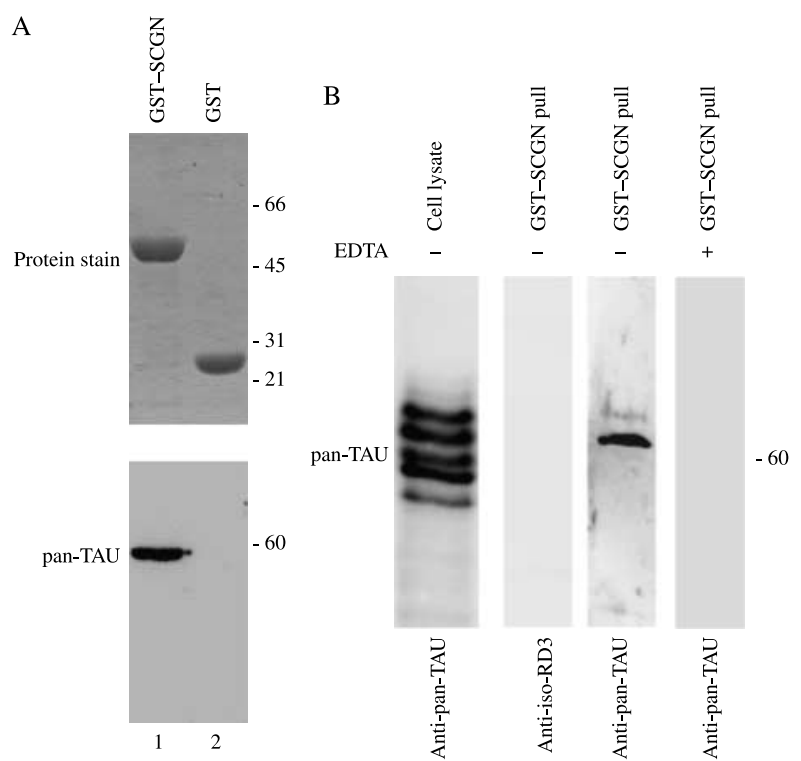

Figure 5 Recombinant SCGN and TAU interaction. The recombinant fusion protein GST-SCGN was loaded onto gluthathionesepharose beads (upper panel, lane 1) and as control GST alone (upper panel, lane 2). Both variants were incubated in Rin-5F cell lysate, and bound protein was eluted from its binding partner after washing off non-specific interacting proteins. The eluate was loaded onto SDS-PAGE, blotted onto nitrocellulose and developed for the presence of TAU using a pan-TAU-specific antibody (lower panel, lane 1 derived from GST-SCGN; lane 2 derived from GST alone). (B) Gluthathione-sepharose beads loaded with GST-SCGN were exposed to Rin-5F cell lysate in the absence (lane 2,3) and in the presence of $5 \mathrm{mM}$ EDTA (lane 4). The eluate of washed beads was loaded on SDS-PAGE, blotted onto nitrocellulose and developed for 3R TAU variants (lane 2) or for pan-TAU (lane 1, 3, 4). Cell lysate input (lane 1).

various concentrations in the cell extract/binding buffer. This clearly eliminated SCGN and TAU interaction even at the lowest tested EDTA concentrations $(2.5 \mathrm{mM})$, indicating the importance of calcium for the TAU-SCGN interaction (Fig. 5B, lane 4).

\section{Comparative MTT assay in Rin-5F clones}

Calcium-binding proteins are known to exert cell protective effects whereas high TAU levels are observed in neurodegenerative disorders. For that reason, we evaluated by MTT assay whether Rin-5F clones exhibiting different SCGN and TAU expression intensities vary in their susceptibility to stress-induced cell death and changes in proliferation.

Clone 1 , which was characterized by the highest amounts of TAU levels and the presence of TAU aggregates (see Fig. 1B, upper panel), is the most sensitive to stress conditions when compared to clone $2(\star P<0 \cdot 0001)$, clone $3-$ control $(\star P<0 \cdot 004)$ and clone $4(\star P<0 \cdot 0001$; Fig. 6). Demonstrated is the mean \pm S.D. of quadruplicate samples of one representative experiment out of three.

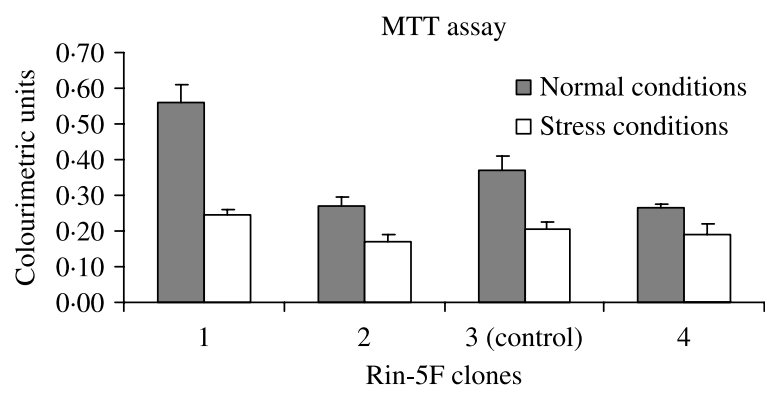

Figure 6 MTT assay for Rin-5F clones. MTT assay upon exposure of Rin-5F clones to low protein concentration (stress condition) for $24 \mathrm{~h}$ compared with complete culture medium. The highest effect on apoptosis and growth inhibition was observed in clone 1 $(-0 \cdot 30 \pm 0.06$ colourimetric units), which was compared to clones $2(-0 \cdot 10 \pm 0.03$ colourimetric units; $P<0 \cdot 0007)$, clone 3 - control $(-0 \cdot 16 \pm 0.02$ colourimetric units; $P<0.004)$ and clone $4(-0.08 \pm 0.02$ colourimetric units; $P<0.0003)$.

\section{Discussion}

Neurodegenerative diseases and the progressive loss of $\beta$-cell function occurring in the course of diabetes mellitus type 2 share pathophysiological characteristics. Moreover, neurons and neuroendocrine $\beta$-cells share several physiological properties, especially with respect to secretory processes. In $\beta$-cells, little is known about the expression of TAU, a key mediator of neurodegeneration with potential involvement in secretion. In this study, we demonstrated the expression of TAU and its isoforms in pancreatic $\beta$-cells, in insulinoma tissue as well as in the rat insulinoma cell lines Rin-5F and INS-1E.

An interesting issue regarding protection against neurodegeneration is EF-hand calcium-binding protein, which exerts positive effects (Hof et al. 1993, Greene et al. 2001). The importance of calcium-binding proteins for TAU dynamics in the CNS was highlighted by Vega et al. (2008). In this respect, it is important to state that the hexa EF-hand calcium-binding protein SCGN is highly and specifically expressed in pancreatic $\beta$-cells and insulinomas (Wagner et al. 2000, Cras-Meneur et al. 2004). Important in this direction was the observation that pyramidal neurons in the hippocampus expressing SCGN are devoid of hyperphosphorylated TAU. This motivated us to study whether SCGN and TAU can interact in insulinoma cells and might fulfil a similar task at neuroendocrine sites. Thereby, we conducted pull-down assays and demonstrated a calcium-dependent interaction of one TAU isoform with SCGN. Following this observation, we looked at SCGN overexpressing Rin-5F clones whether this protein might influence the biophysical state of TAU and $\beta$-cell degenerative effects. This was tested by the proliferation/apoptosis sensitive MTT assay. Higher extent of SCGN overexpression did not provide neuroprotection when TAU aggregates were present. About moderate SCGN overexpression, no clear conclusion could be reached within the border of this study. A higher number of clones have to be tested in further investigations. 
We demonstrated high expression levels of various TAU isoforms in rodent insulinoma cells. The morphological appearance of TAU in the cytoplasm of insulin-secreting cells and its comparison with $\boldsymbol{\alpha}$-tubulin in dual-colour confocal microscopy support the assumption that microtubule dynamics and TAU are of similar importance in the secretory process of neurons (Goedert \& Jakes 1990) and neuroendocrine cells via influencing the axoplasmic transport (LaPointe et al. 2009). For the first time, we elucidated TAU isoform size pattern in $\beta$-cell-derived cell lines. Two larger size variants are absent in the CNS when comparing $\beta$-cell-derived isoforms with that of mammalian brain. Size variants different from CNS have been described in peripheral neurons (Taleghany \& Oblinger 1992, Boyne et al. 1995) earlier.

Furthermore, we showed in this study that TAU aggregates can be found in insulinomas and insulinoma cell lines by means of cytoplasmic deposits as well as in terms of smears by size fractionation in SDS-PAGE followed by TAU-specific immunoblots. In diabetes mellitus type 2 , it has already been shown by immunohistological studies that P-TAU can be seen in islets (Miklossy et al. 1999, 2008, Janson et al. 2004). We showed in addition by phosphoblotting, density gradient sedimentation, and sarkosylate treatment of cell lysate that aggregates can be found at various quantities depending on the selected Rin-5F clone.

Protein phosphorylation is tightly regulated in pancreatic $\beta$-cells by intracellular calcium fluctuations. Each peak of insulin secretion is preceded by an intracellular calcium spike (Gilon et al. 1993). This rise in calcium is necessary for the activation of motor proteins and granule expulsion. SCGN represents an important calcium sensor protein (Rogstam et al. 2007) in $\beta$-cells and might therefore be a candidate for signal transduction.

SCGN has been shown to have a specific expression pattern (Wagner et al. 2000, Gartner et al. 2001, BirkenkampDemtroder et al. 2005, Lai et al. 2006, Attems et al. 2007). Especially, the islets of Langerhans are known for highest levels of SCGN expression (Cras-Meneur et al. 2004). The co-expression of SCGN and TAU in Rin-5F cells with overlapping intracellular distribution implicated association of both proteins, which was confirmed by pull-down assays. Interestingly, our results showed that the association of SCGN is restricted to the $4 \mathrm{R}$ isoform. This variant seems to play an important role in neurodegeneration (Probst et al. 2000). We demonstrated that the calcium-binding protein SCGN interacts with this TAU isoform in a calcium-dependent mode, which might point towards its contribution to the fine tuning of tubule dynamics with impact on insulin secretion.

To investigate whether similar features of tauopathy such as in $\mathrm{AD}$ brains might occur in insulin-secreting cells, we screened these cells for the presence of P-TAU and sarkosylinsoluble TAU aggregates. Indeed, we found a considerable amount of P-TAU and sarkosyl-insoluble TAU in Rin-5F cells. Using disrupted Rin-5F cells, we performed sucrose density gradient centrifugation and demonstrated that TAU co-distributes with tubulin and SCGN in cytosolic fractions of $\sim 0 \cdot 3 \mathrm{M}$. However, at higher densities, TAU was detected in the absence of tubulin. TAU found in $\sim 0.8 \mathrm{M}$ sucrose fractions might represent aggregates, which is supported by TAU appearance in LD and HD using differential ultracentrifugation. This finding has some resemblance to AD brain lysate sucrose fractionation, where much of TAU was found in heavy membrane fractions together with the membrane marker SNAP-25 (personal observation).

Possibly TAU aggregates detected in high-density sucrose fractions of Rin- $5 \mathrm{~F}$ cells are in line with the observation that insulinoma cell lines contain sarkosyl-insoluble TAU. To further confirm this assumption, the sarkosyl-insoluble pellet was dissolved in SDS buffer and then layered onto the same sucrose gradient similarly as described by previous authors (Ksiezak-Reding et al. 1994). This revealed that some of the TAU protein could not be dissolved in SDS into light molecules such as it was seen for SCGN. SCGN equivocally appeared in the light fractions after SDS treatment (M Maj, W Gartner, A Ilhan, D Neziri, J Attems, L Wagner, unpublished observation). The co-appearance of TAU and SCGN in the sarkosyl-insoluble pellet suggests aggregation of SCGN probably together with TAU but this is not irreversible such as it can be seen for TAU. In this respect, it is plausible to speculate that certain TAU mutations which are known to trigger tauopathies (Goedert \& Spillantini 2006) have impact on $\beta$-cell function via impaired microtubule dynamics (Levy et al. 2005).

In analogy to the neurons in $\mathrm{AD}$, increased deposits of TAU and $\beta$-amyloid are found in $\beta$-cells of patients suffering from diabetes mellitus type 2 (Miklossy et al. 2008). In order to evaluate the impact of diabetic conditions on TAU expression, we exposed Rin-5F cells to sera obtained from diabetic patients. This resulted in a marginally increased TAU expression. At this point, it has to be stressed that the manifestation of diabetes mellitus type 2 is preceded by several years of hyperinsulinaemia and prediabetic states. Thus, the trend towards an increased expression of TAU in Rin- $5 \mathrm{~F}$ cells exposed to serum obtained from diabetic patients, despite the lack of statistical significance, might be of biological relevance.

In summary, we described the presence of six TAU isoforms in insulin-producing cells. Furthermore, we showed TAU protein appearance in hyperphosphorylated states as well as in protein deposits and as sarkosyl-insoluble aggregates, which all are resembling features of tauopathy in AD. Additionally, we demonstrated co-expression and association of one TAU isoform and the calcium-binding protein SCGN in insulin-secreting cells. Owing to the bivalent intracellular functions of TAU proteins and the calcium dependency of the TAU-SCGN interaction, it seems plausible that these findings have an impact on the physiology of insulin secretion as well as on pathological processes leading to $\beta$-cell destruction.

\section{Supplementary data}

This is linked to the online version of the paper at http://dx.doi.org/10.1677/ JOE-09-0341. 


\section{Declaration of interest}

The authors declare that there is no conflict of interest that could be perceived as prejudicing the impartiality of the research reported.

\section{Funding}

This work was supported by a research grant 'Major of city of Vienna Fond', number 08052

\section{Acknowledgements}

We thank Prof. Anton Luger (Medical University of Vienna) and Prof. J H Nielsen (University of Copenhagen) for support.

\section{References}

Albrektsen T, Richter HE, Clausen JT \& Fleckner J 2001 Identification of a novel integral plasma membrane protein induced during adipocyte differentiation. Biochemical Journal 359 393-402.

Alonso AD, Zaidi T, Novak M, Barra HS, Grundke-Iqbal I \& Iqbal K 2001 Interaction of tau isoforms with Alzheimer's disease abnormally hyperphosphorylated tau and in vitro phosphorylation into the disease-like protein. Journal of Biological Chemistry 276 37967-37973.

Attems J, Quass M, Gartner W, Nabokikh A, Wagner L, Steurer S, Arbes S, Lintner F \& Jellinger K 2007 Immunoreactivity of calcium binding protein secretagogin in the human hippocampus is restricted to pyramidal neurons. Experimental Gerontology 42 215-222.

Attems J, Preusser M, Grosinger-Quass M, Wagner L, Lintner F \& Jellinger K 2008 Calcium-binding protein secretagogin-expressing neurones in the human hippocampus are largely resistant to neurodegeneration in Alzheimer's disease. Neuropathology and Applied Neurobiology 34 23-32.

Birkenkamp-Demtroder K, Wagner L, Brandt Sorensen F, Bording Astrup L, Gartner W, Scherubl H, Heine B, Christiansen P \& Orntoft TF 2005 Secretagogin is a novel marker for neuroendocrine differentiation. Neuroendocrinology 82 121-138.

Boyne LJ, Tessler A, Murray M \& Fischer I 1995 Distribution of big tau in the central nervous system of the adult and developing rat. Journal of Comparative Neurology 358 279-293.

Cras-Meneur C, Inoue H, Zhou Y, Ohsugi M, Bernal-Mizrachi E, Pape D, Clifton SW \& Permutt MA 2004 An expression profile of human pancreatic islet mRNAs by serial analysis of gene expression (SAGE). Diabetologia 47 284-299.

Delacourte A \& Defossez A 1986 Alzheimer's disease: tau proteins, the promoting factors of microtubule assembly, are major components of paired helical filaments. Journal of Neurological Sciences 76 173-186.

Dias WB \& Hart GW 2007 O-GlcNAc modification in diabetes and Alzheimer's disease. Molecular BioSystems 3 766-772.

Gartner W, Lang W, Leutmetzer F, Domanovits H, Waldhausl W \& Wagner L 2001 Cerebral expression and serum detectability of secretagogin, a recently cloned EF-hand $\mathrm{Ca}(2+)$-binding protein. Cerebral Cortex $\mathbf{1 1}$ 1161-1169.

Gartner W, Vila G, Daneva T, Nabokikh A, Koc-Saral F, Ilhan A, Majdic O, Luger A \& Wagner L 2007 New functional aspects of the neuroendocrine marker secretagogin based on the characterization of its rat homolog. American Journal of Physiology. Endocrinology and Metabolism 293 E347-E354.

Gilon P, Shepherd RM \& Henquin JC 1993 Oscillations of secretion driven by oscillations of cytoplasmic $\mathrm{Ca}^{2+}$ as evidences in single pancreatic islets. Journal of Biological Chemistry 268 22265-22268.

Goedert M \& Jakes R 1990 Expression of separate isoforms of human tau protein: correlation with the tau pattern in brain and effects on tubulin polymerization. EMBO Journal 9 4225-4230.
Goedert M \& Spillantini MG 2006 A century of Alzheimer's disease. Science 314 777-781.

Goedert M, Wischik CM, Crowther RA, Walker JE \& Klug A 1988 Cloning and sequencing of the cDNA encoding a core protein of the paired helical filament of Alzheimer disease: identification as the microtubule-associated protein tau. PNAS $\mathbf{8 5}$ 4051-4055.

Greenberg SG \& Davies P 1990 A preparation of Alzheimer paired helical filaments that displays distinct tau proteins by polyacrylamide gel electrophoresis. PNAS 87 5827-5831.

Greene JR, Radenahmad N, Wilcock GK, Neal JW \& Pearson RC 2001 Accumulation of calbindin in cortical pyramidal cells with ageing; a putative protective mechanism which fails in Alzheimer's disease. Neuropathology and Applied Neurobiology 27 339-342.

Grundke-Iqbal I, Iqbal K, Tung YC, Quinlan M, Wisniewski HM \& Binder LI 1986 Abnormal phosphorylation of the microtubule-associated protein tau (tau) in Alzheimer cytoskeletal pathology. PNAS 83 4913-4917.

Hof PR, Nimchinsky EA, Celio MR, Bouras C \& Morrison JH 1993 Calretinin-immunoreactive neocortical interneurons are unaffected in Alzheimer's disease. Neuroscience Letters 152 145-148.

Janson J, Ashley RH, Harrison D, McIntyre S \& Butler PC 1999 The mechanism of islet amyloid polypeptide toxicity is membrane disruption by intermediate-sized toxic amyloid particles. Diabetes 48 491-498.

Janson J, Laedtke T, Parisi JE, O’Brien P, Petersen RC \& Butler PC 2004 Increased risk of type 2 diabetes in Alzheimer disease. Diabetes 53 474-481.

Khlistunova I, Biernat J, Wang Y, Pickhardt M, von Bergen M, Gazova Z, Mandelkow E \& Mandelkow EM 2006 Inducible expression of tau repeat domain in cell models of tauopathy: aggregation is toxic to cells but can be reversed by inhibitor drugs. Journal of Biological Chemistry 281 1205-1214.

Kosik KS, Joachim CL \& Selkoe DJ 1986 Microtubule-associated protein tau (tau) is a major antigenic component of paired helical filaments in Alzheimer disease. PNAS 83 4044-4048.

Ksiezak-Reding H, Morgan K \& Dickson DW 1994 Tau immunoreactivity and SDS solubility of two populations of paired helical filaments that differ in morphology. Brain Research 649 185-196.

Lai M, Lu B, Xing X, Xu E, Ren G \& Huang Q 2006 Secretagogin, a novel neuroendocrine marker, has a distinct expression pattern from chromogranin A. Virchows Archiv 449 402-409.

LaPointe NE, Morfini G, Pigino G, Gaisina IN, Kozikowski AP, Binder LI \& Brady ST 2009 The amino terminus of tau inhibits kinesin-dependent axonal transport: implications for filament toxicity. Journal of Neuroscience Research 87 440-451.

Levy SF, Leboeuf AC, Massie MR, Jordan MA, Wilson L \& Feinstein SC 2005 Three- and four-repeat tau regulate the dynamic instability of two distinct microtubule subpopulations in qualitatively different manners. Implications for neurodegeneration. Journal of Biological Chemistry 280 13520-13528.

Lorenzo A, Razzaboni B, Weir GC \& Yankner BA 1994 Pancreatic islet cell toxicity of amylin associated with type-2 diabetes mellitus. Nature $\mathbf{3 6 8}$ 756-760.

Miklossy J, Taddei K, Martins R, Escher G, Kraftsik R, Pillevuit O, Lepori D \& Campiche M 1999 Alzheimer disease: curly fibers and tangles in organs other than brain. Journal of Neuropathology and Experimental Neurology $\mathbf{5 8}$ 803-814.

Miklossy J, Qing H, Radenovic A, Kis A, Vileno B, Laszlo F, Miller L, Martins RN, Waeber G, Mooser V et al. 2008 Beta amyloid and hyperphosphorylated tau deposits in the pancreas in type 2 diabetes. Neurobiology of Aging. DOI: 10.1016/j.neurobiolaging.2008.08.019.

O'Brien TD, Butler PC, Kreutter DK, Kane LA \& Eberhardt NL 1995 Human islet amyloid polypeptide expression in COS-1 cells. A model of intracellular amyloidogenesis. American Journal of Pathology 147 609-616.

O'Rahilly S, Turner RC \& Matthews DR 1988 Impaired pulsatile secretion of insulin in relatives of patients with non-insulin-dependent diabetes. New England Journal of Medicine 318 1225-1230.

Probst A, Gotz J, Wiederhold KH, Tolnay M, Mistl C, Jaton AL, Hong M, Ishihara T, Lee VM, Trojanowski JQ et al. 2000 Axonopathy and amyotrophy in mice transgenic for human four-repeat tau protein. Acta Neuropathologica 99 469-481. 
Ristow M 2004 Neurodegenerative disorders associated with diabetes mellitus. Journal of Molecular Medicine 82 510-529.

Rogstam A, Linse S, Lindqvist A, James P, Wagner L \& Berggard T 2007 Binding of calcium ions and SNAP-25 to the hexa EF-hand protein secretagogin. Biochemical Journal 401 353-363.

Santacruz K, Lewis J, Spires T, Paulson J, Kotilinek L, Ingelsson M, Guimaraes A, DeTure M, Ramsden M, McGowan E et al. 2005

Tau suppression in a neurodegenerative mouse model improves memory function. Science 309 476-481.

Schubert D, Behl C, Lesley R, Brack A, Dargusch R, Sagara Y \& Kimura H 1995 Amyloid peptides are toxic via a common oxidative mechanism. PNAS 92 1989-1993.

Simpson IA, Yver DR, Hissin PJ, Wardzala LJ, Karnieli E, Salans LB \& Cushman SW 1983 Insulin-stimulated translocation of glucose transporters in the isolated rat adipose cells: characterization of subcellular fractions. Biochimica et Biophysica Acta 763 393-407.

Taleghany N \& Oblinger MM 1992 Regional distribution and biochemical characteristics of high molecular weight tau in the nervous system. Journal of Neuroscience Research 33 257-265.
Tischler AS 2002 Chromaffin cells as models of endocrine cells and neurons. Annals of the New York Academy of Sciences 971 366-370.

Vega IE, Traverso EE, Ferrer-Acosta Y, Matos E, Colon M, Gonzalez J, Dickson D, Hutton M, Lewis J \& Yen SH 2008 A novel calcium-binding protein is associated with tau proteins in tauopathy. Journal of Neurochemistry 106 96-106.

Wagner L, Oliyarnyk O, Gartner W, Nowotny P, Groeger M, Kaserer K, Waldhausl W \& Pasternack MS 2000 Cloning and expression of secretagogin, a novel neuroendocrine- and pancreatic islet of Langerhans-specific $\mathrm{Ca}^{2+}$-binding protein. Journal of Biological Chemistry $27524740-24751$.

Received in final form 23 December 2009

Accepted 8 January 2010

Made available online as an Accepted Preprint

8 January 2010 